\title{
The Use of Wavelets to Identify Frequency Hopped Signals
}

\author{
Ralph Hippenstiel, Nabil Khalil, and Monique Fargues \\ Electrical and Computer Engineering Department \\ Naval Postgraduate School \\ Monterey, CA 93943 \\ hippenst@ece.nps.navy.mil
}

\begin{abstract}
Digital signal processing is used in the interception of digital communication signals. Due to the inherent security features, frequency hopped signals $(\mathrm{FH})$ are widely used in both military and commercial communication applications. The interception of $\mathrm{FH}$ signals can be addressed in several ways. In this work we use the Instantaneous Correlation Function (ICF) to represent non-stationary signals. We relate the wavelet transform the ICF surface of a signal to its Wigner-Ville Distribution (WVD). The signals can be observed in the scale surfaces of the wavelet domain. An energy analysis is applied to the surfaces (in the wavelet domain) to identify the scale of the FH signal and to estimate the hop frequency. The FH signals can also be identified by inspecting the pattern of the scales of a multiple-hop-observation interval. If all hop frequencies are within one wavelet scale, then the FH signal can be identified by a set of distinct hop frequencies.
\end{abstract}

\section{Introduction}

In the open literature, the interception of digital communication signals is one of the applications of digital signal processing. Primarily due to the inherent security features of frequency hopped signals $(\mathrm{FH})$ they are widely used in both military and commercial communication applications. The interception of FH signals can be addressed in several ways. Typical examples of identification and estimation procedures are the wide band or channelized receiver, the Wigner-Ville type time-frequency Distribution, and cyclostationary processing. Wavelet processing promises computational advantages. In this work we address the use of the Instantaneous Correlation Function (ICF) to represent non-stationary signals. We relate the wavelet transform the ICF surface of a signal to its Wigner-Ville Distribution (WVD). The signals can be observed in the scale surfaces in the wavelet domain. An energy analysis can be applied to the surfaces (in the wavelet domain) to identify the scale of the FH signal and to estimate the hop frequency. The FH signals can also be identified by inspecting the pattern of the scales of a multiple-hop-observation interval. If only one wavelet scale covers the hop frequency interval, then the FH signal can be identified by the diversity of hop frequencies.

\section{Instantaneous Correlation Functions}

There are two widely used expressions for correlation functions. The first one uses the averaging operator, in form of integration or expectation operators and typically defines the correlation functions of stationary signals. The integration operator is applied to deterministic signals while the expectation operator is applied to stochastic signals. The second expression does not apply averaging and is mainly used for non-stationary signals. It typically uses the product of two time samples. We use this concept in the Instantaneous Correlation Function (ICF) of the nonstationary signal $x(t)$ which is defined as

$$
R_{x}^{i}(t, \tau)=x\left(t+\frac{\tau}{2}\right) x^{*}\left(t-\frac{\tau}{2}\right),
$$

where " $i$ " stands for instantaneous feature, " $t$ " " is the time and " $\tau$ "" is the time delay (lag). The ICF is conjugate symmetric with respect to the time delay, i.e., $R_{x}^{i}(t, \tau)=R_{x}^{i *}(t,-\tau)$. Also, at $\tau=0 R_{x}^{i}(t, 0)=$ $|x(t)|^{2}$, the instantaneous power of $x(t)$ at time $t$.

\section{Wavelet Transforms of the Instantaneous Correlation Functions}

As far as the time and frequency resolution is concerned, the wavelet transform (WT) has a significant 
advantage relative to the Fourier transform. The wavelet analysis applies a band pass filter at each scale " $s$ ". All filters have a constant ratio of bandwidth to center frequency (i.e., constant $Q$ filter). The wavelet analysis may be also seen as an expansion (projection or inner product) using a set of localized (finite support) basis functions. These basis function typically form a complete orthonormal set. All of the basis function can be obtained by scaling and translating the so called mother wavelet. The continuous time wavelet transform of the finite energy signal $x(t)$ is given by $[1,2]$

$$
W_{x}(s, \ell)=\frac{1}{\sqrt{s}} \int_{-\infty}^{\infty} x(t) g^{*}\left(\frac{t-\ell}{s}\right) d t
$$

where $g(t)$ is the mother wavelet function which has been scaled by wavelet scale " $s$ " " and shifted to " $\ell$ " " over the time variable axis " $t$ "". In this transformation the time variable " $t$ "" has been substituted by two wavelet domain variables " $s$ " and " $\ell$ " " giving the representation of $x(t)$ over the wavelet co-domain (i.e., the time-scale plane) [2]. The discrete wavelet transform represents the signal $x(t)$ as

$$
x(t)=\sum_{k=-\infty}^{\infty} c(k) \phi_{k}(t)+\sum_{j=0}^{\infty} \sum_{k=-\infty}^{\infty} d(j, k) \psi_{(j, k)(t)}
$$

where $j$ and $k$ are integers, the coefficient $c(k)$ constitutes the coefficients of the approximation, while $d(j, k)$ constitutes the coefficients of the added details or equivalently the fine resolutions. If the wavelets and the associated scaling functions form an orthonormal set of basis functions the coefficients are given by

$$
c(k)=\left\langle f(t), \phi_{k}(t)\right\rangle,
$$

and

$$
d(j, k)=\left\langle f(t), \psi_{j, k}(t)\right\rangle .
$$

Usually we choose short (finite support) wavelets.

\section{WT of the Instantaneous Correlation Function}

The Wigner-Ville Distribution (WVD) of the signal $\mathrm{x}(\mathrm{t}), V_{x}(t, f)$, is related to the wavelet transform of the ICF of $\mathrm{x}(\mathrm{t}), W_{x x}^{i}(t ; s, \ell)$ as [3]

$$
W_{x x}^{i}(t ; s, \ell)=\sqrt{s} \int_{-\infty}^{\infty} G^{*}(s f) V_{x}(t, f) e^{j 2 \pi f \ell} d f
$$

where $V_{x}(t, f)=W V D(x(t))$.

This equation relates $W_{x x}^{i}(t ; s, \ell)$ at any wavelet scale $s$ to the WVD of $V_{x}(t, f)$ and suggests that we can obtain a filtered version of the WVD by Fourier transforming $W_{x x}^{i}(t ; s, \ell)$. This relation enables us to cxploit the wavelet transform of the ICF surface to obtain filtered samples of the WVD of the original FH signal. The WVD samples are obtained by a Fourier transform and can be used to extract the frequency of the original FH signal (the hop froquency).

\section{Interception of Frequency Hopped Signals}

Spread spectrum signals are a widely used digital modulation technique. The frequency hopped (FH) signals are a subset of spread spectrum modulations. The FH scheme can use one of two modulation techniques. The first is the data base band modulation, and the second is the spreading technique. The most widely used FH scheme uses M-ary Frequency Shift Keying modulation as a the data base band modulation. This FH system is known as pure FH scheme. For the pure FH signal, it is shown in [3] that the ICF surface has a cellular (diamonds) structure over the time and time delay plane.

Consider the wavelet transform of the ICF surface with respect to the time delay variable. The wavelet transform will generate a 3 -D surface (i.e., time, wavelet shift, and wavelet scale). A 2-D surface is used for each wavelet scale of interest. Identifying FH signals from 2-D surfaces of the wavelet domain is an application of wavelet processing to the correlation function. We address the interception identification problem in two steps. First, we inspect the 2-D surfaces to identify and classify the structure of the FH signal and obtain a rough estimate for the hop time interval. Then, using the hop time intervals we can apply an energy analysis to each hop at all wavelet scales of interest. The energy analysis assigns a scale index (called the proper scale) to each hop. The proper scale, for each hop, is that scale which has the greatest energy content. The sequence of proper scales, representing the hop sequence, is called the hop-scale pattern. Then, the frequency of each hop can be extracted at the proper scale.

If the monitored signal is sampled such that it will be contained in two or more wavelet scales, then the hop-scale pattern of the $\mathrm{FH}$ signal is different from the hop-scale patterns of other digital modulation signals. If all frequency hops are contained in one scale then a large set of hopping frequencies will be the distinctive feature of the $\mathrm{FH}$ signal. The automated extraction of the hop time intervals is part of the interception procedure. We approach this problem using an edge detection approach which applies an edge detection operator, well known in image processing, over the wavelet domain surfaces. 


\subsection{Scale Identification}

Parseval's equality theorem for the complete orthogonal filter banks (for L itcrations) [3], is given by

$$
\|x(n)\|^{2}=\sum_{k \in Z}\left(|C(L, 2 k)|^{2}+\sum_{j=1}^{L}|d(j, 2 k+1)|^{2}\right)
$$

where, in the sense of wavelet analysis, $C(L, 2 k)$ are the scaling coefficients at the scale $L, d(j, 2 k+1)$ are the wavelet coefficients at scale $j$, and $k$ is the wavelet shift variable. The average energy per wavelet coefficient at the scale $j$ is given by

$$
A(j)=\sum_{k \in Z} \sum_{j=1}^{L}|d(j, 2 k+1)|^{2} / N(j)
$$

where $N(j)$ is the number of wavelet coefficients at the scale $j$. The maximum $A(j)$ identifies the (proper) scale of the underlying hop.

\subsection{Hop Interval Extraction}

Since the resultant wavelet surfaces have a cellular (i.e., diamond) structure (see Fig. 1), we can apply the so called compass operator [4] to detect the edges of the diamond structure. Figure 1 shows the ICS and the first 5 scale outputs for a staircase frequency hopped signal. The edges are characterized by a fixed inclination degree relative to the time axis. If the compass operator is dedicated to this angle $\left( \pm 45^{\circ}\right)$, the edges can be detected and the intersection with the time axis determines the hop start (or stop) point.

\subsection{Hop Frequency Extraction}

We can exploit equation (6) by applying one Fourier transform, in the center of the hop diamond over the wavelet domain to obtain a spectral estimate of the FH signal for the underlying hop. The scale index points to the (proper) scale where the maximum signal energy is located, allowing a spectral estimate at the best possible SNR.

\section{Processing Scheme, Simulations and Results}

\subsection{Processing Scheme}

The processing scheme is set up is as follows: (1) Compute the analytic form of the monitored signal and compute its ICF surface. (2) Compute the discrete time wavelet analysis of the ICF surface with
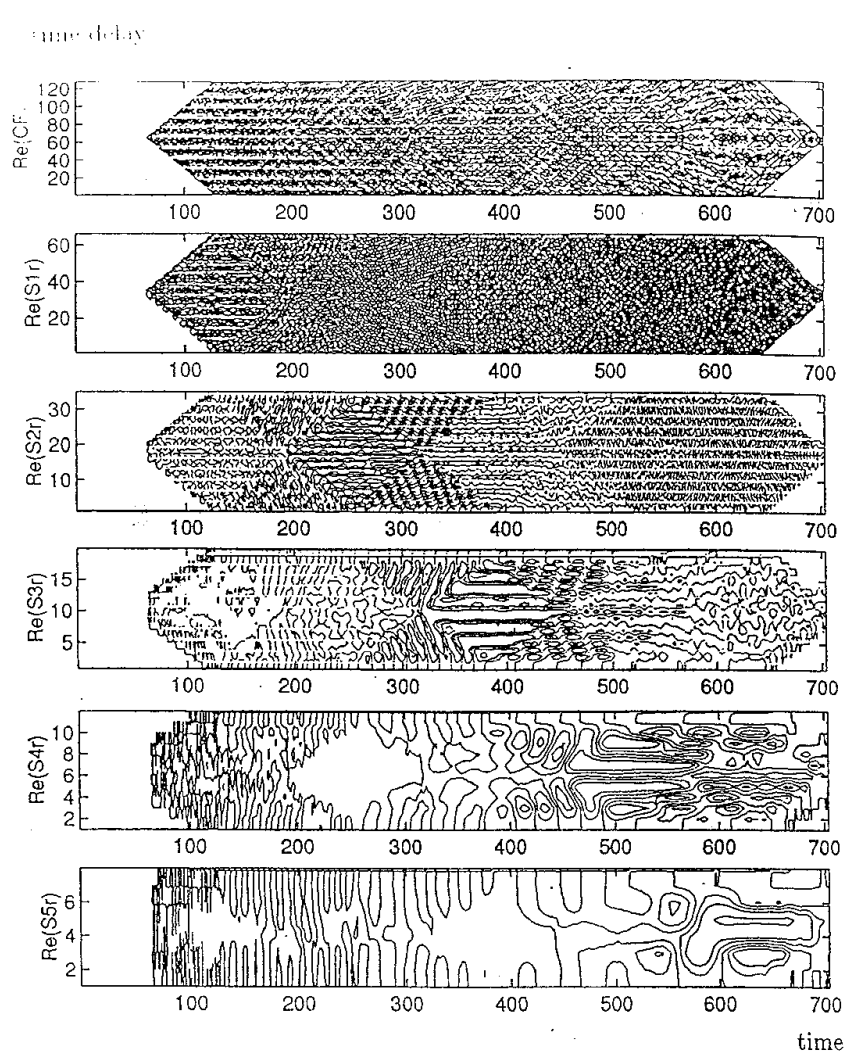

Figure 1: Wavelet surfaces of FH signals using Daub-3 at $10 \mathrm{~dB}$ (scale 1-5).

respect to the time delay. The suggested number of scales is the number of octaves in the monitored band of frequencies. (3) Based on the estimated hop time intervals (or a priori, information), carry out the energy analysis to identify the proper scale. Then, extract the hop times and the hop frequency from the wavelet surface at the proper scale.

\subsection{Performance Evaluation}

Performance of the scale identification is given to the probability of success, $P_{i d}$, and is defined as

$$
P_{i d}=\frac{\text { the number of correctly identified scales }}{\text { the total number of hops }}
$$

where the probability of error is $P_{e}=1-P_{i d}$ accounts for the probability of false alarm and the probability of a miss. Performance of the frequency estimation is given by the probability of success, $P_{f}$, and is defined as

$P_{f}=\frac{\text { the number of correctly extracted frequencies }}{\text { the total number of hops }}$ 
where the frequency is said to be correctly identified if its value lies within \pm one bin of the Fourier transform. The mumber of Fourier bins corresponds to the number of samples over the duration of one hop. Performance of the hop time estimation is given by the mean squared error of the hop time interval, $T_{H}$.

\subsection{Simulation Results}

Test data was created having four hop frequencies $[24,12,6,3]$. This set spans four wavelet scales at sampling frequency of 128 . Each signal has five hops (i.e., one hop repeats). Different realizations are obtained at signal to noise ratios (SNR) $[10,6,3,-3,-6]$ $\mathrm{dB}$. At each SNR, 10 realizations are generated. The noise is white Gaussian noise. The MATLAB Wavelet Toolbox was used for wavelet analysis. Standard Daubechies wavelet basis functions are applied with lengths 4, 8, 16, 32. Figure 2 shows the scale success rate $P_{i d}$ versus different SNRs for different wavelet scales. Figure 3 shows the frequency success rate $P_{f}$ versus different SNRs for different wavelet scales. Figure 4 shows the mean squared error of the hop duration estimation error. The standard deviation is plotted in data points versus SNR, where the true hop duration is 128 points.

\section{Comments and Conclusion}

Intercepting the FH signals from the 2-D surfaces of the wavelet domain is a new application of wavelet processing. Simulation results using Daubechies wavelets of lengths 4,8 , and 16 show that, assuming known hop intervals, a probability of one is achieved for scale identification and frequency extraction of $\mathrm{FH}$ signals at SNRs as low as $0 \mathrm{~dB}$. Although the visual inspection is not addressed in detail here, the complex surface in the wavelet domain is best evaluated when using its real or imaginary value only.

\section{References}

[1] G. Strang and T. Nguyen, Wavelets and Filter Banks, Wellesley-Cambridge Press, New York, 1995.

[2] M. Vetterli and J. Kovacevic, Wavelets and Subband Coding, Prentice-Hall, Inc., Englewood Cliffs, NJ, 1995.

[3] N. Khalil and R. Hippenstiel , "Wavelet Transform of Correlation Function of Frequency Hopped Signals," $30^{\text {th }}$ Asilomar Conf. Signals, Systems and Computers, Vol. 2, Pacific Grove, CA, pp. 964-967, Nov. 3-6, 1996.
4] A. K. JAIN, Fundamentals of Digital Image Processing, Prentice-Hall, Inc., Englewood Cliffs, N.J, 1989.
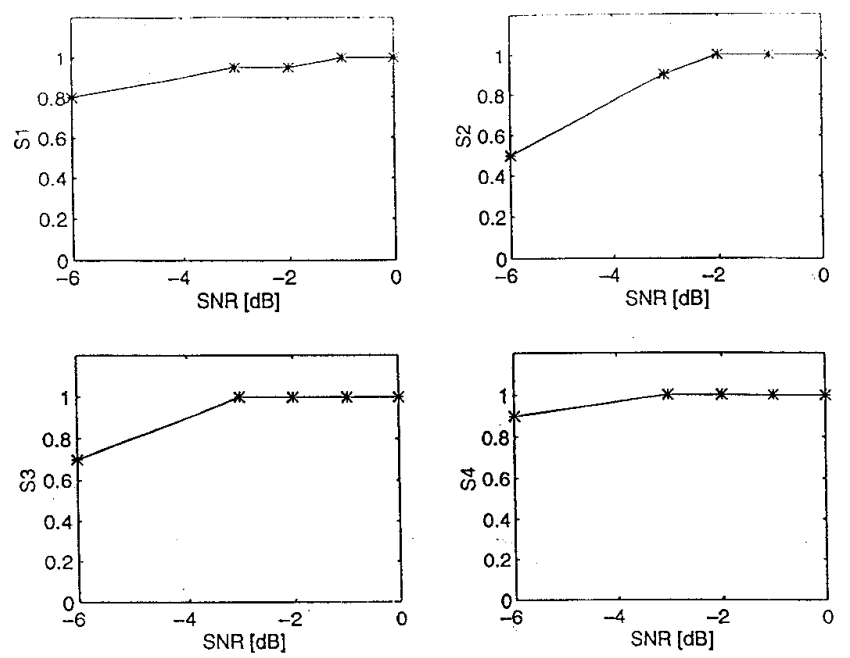

Figure 2: $P_{i} d$ for Daub-8.
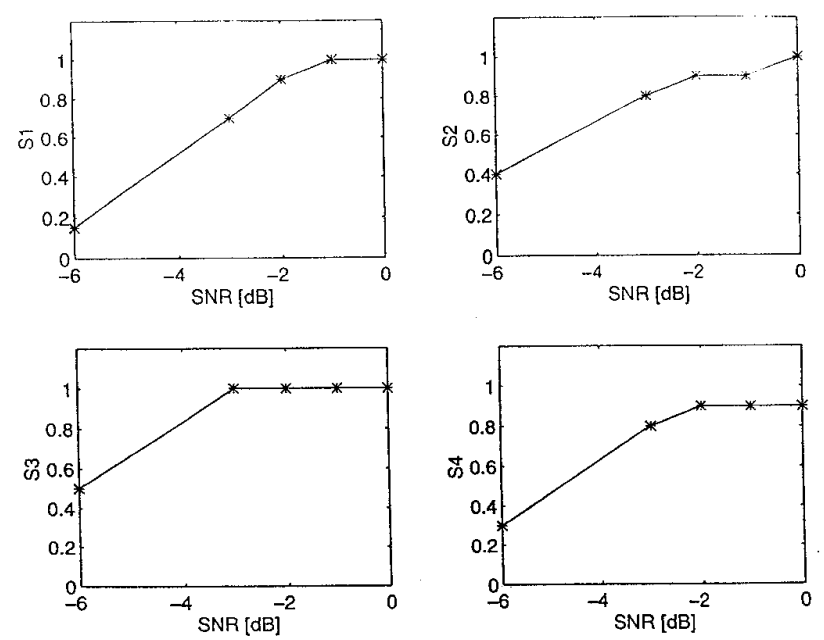

Figure 3: $P_{f}$ for Daub-8.

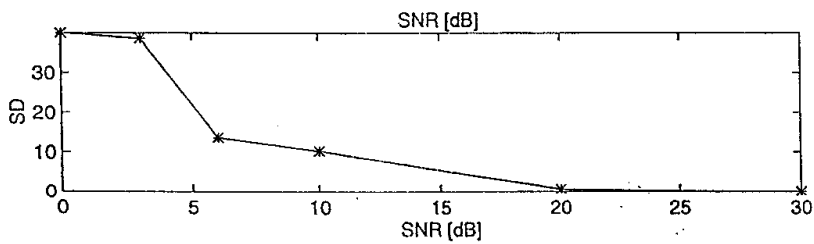

Figure 4: Hop timing estimation error for Daub-8. 